\title{
Feasibility Research of Infinite State Markov Chain Technique in Variable-frequency Speed Control System
}

\author{
Mei Zheng ${ }^{1, a}$, Bo Zheng ${ }^{2, b}$ and Huanhuan Zhang ${ }^{2, c}$ \\ ${ }^{1}$ College of Mechanical \& Electrical engineering, Shaanxi University of Science \& Technology, Xi'an, \\ Shaanxi, China \\ ${ }^{2}$ College of Electrical and Information engineering, Guangxi University of Science and Technology, \\ Liuzhou, Guangxi, China

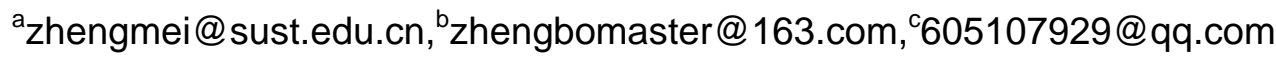

Keywords: SVPWM; Harmonic Wave; Markov Chain; High Frequency Noise

\begin{abstract}
At present,traditional Space Vector Pulse Width Modulation (SVPWM ) technique has been widely used in the variable frequency speed control system,but this technique makes the system produce components of high harmonic wave at integer times of switching frequency.Thus inverter produces conducted Electromagnetic Interference (EMI) and high frequency noise.Faced with these problems,this paper adopts the method which is to add two-state or three-state Markov chain technique to variable frequency speed control system of SVPWM generator to reduce high frequency noise. This method can make amplitude of harmonic components of output current that is produced by inverter of the system effective in reducing and more uniformly distribute in relatively wide frequency range.Through many simulation experiments, this method can effectively reduce amplitude of harmonic components and is more superior than traditional SVPWM technique.
\end{abstract}

\section{Introduction}

Traditional SVPWM technique makes a qualitative leap in variable-frequency speed control technique,but application of traditional SVPWM technique also brings some new problems[1].For example,switching devices of traditional SVPWM technique are turned on and shut off in a certain way,but components of high harmonic wave are produced in the integer times of switching frequency (especially one multiple and two multiple).High harmonic wave led electromagnetic induction motor of system to create a problem which is the high frequency electromagnetic noise.

Regarding of this class of problems, researchers had put forward a method that is random SVPWM technique.Although the method can effectively reduce high harmonic wave,makes equipment structure become simple and effective,can be simply implement,etc[2].But this method also has some problems in real application.Generation of random numbers is basis to implement the random SVPWM technique.Random numbers should absolutely obey uniform distribution in its ideal sense,but it would inevitably produce a phenomenon that continuous multiple random numbers are greater than mathematical expectation or less than mathematical expectation.This phenomenon can make amplitude of high harmonic wave still maintain very high in integral times of switching frequency and cannot be effectively suppressed,so that this method also can increase output ripple current and influence on the control performance of system[3,4].

In view of above problems,the paper putted forward a method that Markov chain technique is applied to SVPWM generator model.Thus the method can make harmonic wave of output current more uniformly distribute in relatively wide frequency range,effectively restrain high frequency noise,make output current waveform become more perfect and reduce ripple current.So that experimental results demonstrate that infinite state Markov chain technique is feasibility and advantage.

\section{Markov Chain Technique}

Markov process is an important branch of stochastic process. So that it has been widely applied to 
many fields.For example,modern physics,biology,management science,information processing, automatic control,computer science,financial insurance and so on [4].

Where $\{X(t), t \in T\}$ is a stochastic process, and $0 \leq t_{1}<t_{2}<\ldots<t_{n} \in \mathrm{T}$.If the values of process $X(t)$ are $x_{1}, X_{2}, \ldots, x_{n-1}$ and $x_{n}$ at these moments $t_{1}, t_{2}, \ldots, t_{n-1}$ and $t_{n}$.Equation of Markov process can be written as Eq.(1):

$$
\mathrm{F}\left(x_{n}, t_{n} \mid x_{n-1}, t_{n-1} ; x_{n-2}, t_{n-2} ; \cdots ; x_{1}, t_{1}\right)=\mathrm{F}\left(x_{n}, t_{n} \mid x_{n-1}, t_{n-1}\right) \text {. }
$$

Markov process also has a special feature that is the stability ineffectiveness theory[5]. When a stochastic process is a Markov process, its stability ineffectiveness means that information of process $x_{1}, x_{2}, \ldots, x_{n-2}$ has all been stored in information of process $x_{n-1}$. Information of process $x_{n}$ can be solved with information of process $x_{n-1}$ and information of process $x_{n}$ has nothing to do with information of process $x_{1}, x_{2}, \ldots, x_{n-2}$. All transition probability of Markov chain can compose a matrix that is called matrix of transition probability.Matrix of transition probability can be written as Eq.(2) by one-step transition probability :

$$
\mathrm{P}=\left\{p_{i j}, i, j \in s=(0,1,2, \cdots, n)\right\}=\left[\begin{array}{cccc}
p_{11} & p_{12} & \cdots & p_{1 j} \\
p_{21} & p_{22} & \cdots & p_{2 j} \\
\vdots & \vdots & \ddots & \vdots \\
p_{i 1} & p_{i 2} & \cdots & p_{i j}
\end{array}\right] .
$$

Each element of matrix $P$ is non-negative number and sum of each row element equals one[5].

Before algorithm of random switching frequency can be written as Eq.(3):

$$
f_{n+1}=f_{s}+s \times R
$$

In Eq.(3), $f_{n+1}$ is frequency of switching function at the time $n+1, f_{s}$ is frequency of rated switching function, $s$ is random number between [-1,1] and $R$ is random gain[6].Random algorithm can make frequency of switching function that according to random regularity distribute within a range $\left[f_{s}-R, f_{s}+R\right]$.

Through algorithm of random switching frequency,Markov chain technique was applied to SVPWM technique base on random switching frequency.State space of Markov chain,in theory,the bigger the better.Along with state space bigger,random numbers would take up more and more resources of processor.In other words,bigger state would make matrix of transition probability has more unknown parameters, so that experimental simulation is not easy to be achieved[7].Synthesizes each kind of situations,feasibility of infinite state Markov chain technique that is applied to variable-frequency speed control system would be proven by simulation results of two-state and three-state Markov chain technique.

Specific design scheme of two-state Markov chain technique as follows:switching frequency is called state one when it is greater than $f_{s}$. Switching frequency is called state two when it is less than $f_{s}$,so that actual mathematical expectation of switching frequency would to equal theoretical mathematical expectation of switching frequency as much as possible[8].Switching state figure of two-state Markov chain is shown in Fig.1.

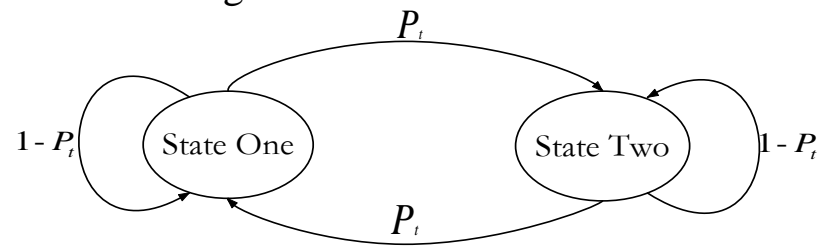

Fig. 1. Switching state figure of two-state Markov chain

As can be seen from Fig.1,if current switching frequency of system belongs to state one, percent probability of next switching frequency that belongs to state two is $p_{t}$. But percent probability of next switching frequency that still belongs to state one is $1-p_{t}$.In a similar way, if current switching 
frequency of system belongs to state two,percent probability of next switching frequency that belongs to state one is $p_{t}$. But percent probability of next switching frequency that still belongs to state two is 1- $p_{t}$.Matrix of transition probability of two-state Markov chain can be written as Eq.(4):

$$
P=\left[\begin{array}{ll}
P_{11} & P_{12} \\
P_{21} & P_{22}
\end{array}\right]=\left[\begin{array}{cc}
1-P_{t} & P_{t} \\
P_{t} & 1-P_{t}
\end{array}\right]
$$

According to matrix of transition probability of two-state Markov chain,simulation model of two-state Markov chain that is derived in the MATLAB/SIMULINK software platform is shown in Fig.2.

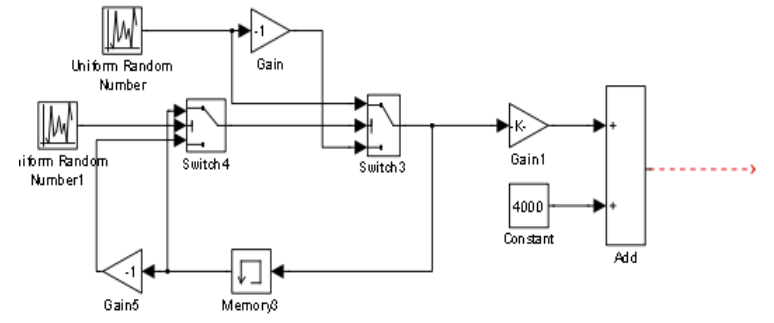

Fig. 2. Simulation model of two-state Markov chain

Specific design scheme of three-state Markov chain technique as follows:change range $\left[f_{s}-R, f_{s}+R\right]$ of switching frequency is divided into three parts $\left[f_{s}-R, f_{s}-k \times R\right],\left[f_{s}-k \times R, f_{s}+k \times R\right]$ and $\left[f_{s}+k \times R, f_{s}+R\right]$, coefficient $k \in(0,0.36)$.In order to further analysis,three change ranges of switching frequency are respectively called state one,state two and state three.Switching state figure of three-state Markov chain is shown in Fig.3.

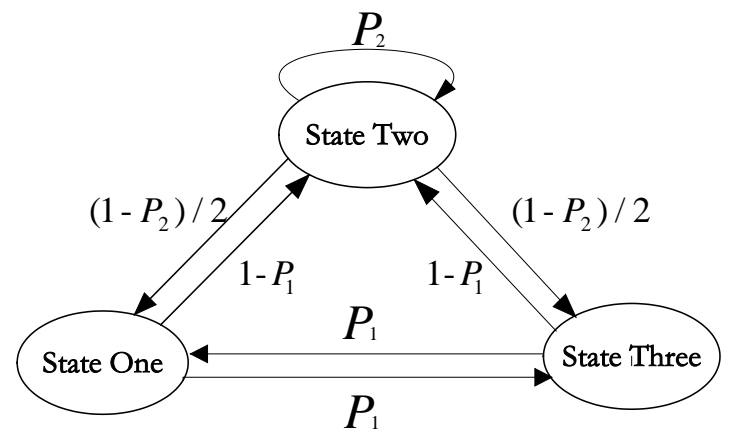

Fig. 3. Switching state figure of three-state Markov chain

As can be seen from switching state diagram figure,if current switching frequency of system belongs to state one,next switching frequency only belongs to state two or state three.Percent probability of next switching frequency that belongs to state two is $1-p_{1}$.Percent probability of next switching frequency that belongs to state three is $p_{1}$.If current switching frequency of system belongs to state two,percent probability of next switching frequency that belongs to state one or three is $\left(1-p_{2}\right) / 2$. But percent probability of next switching frequency that still belongs to state two is $p_{2}$.If current switching frequency of system belongs to state three,next switching frequency only belongs to state one or two.Percent probability of next switching frequency that belongs to state one is $p_{1}$. Percent probability of next switching frequency that belongs to state two is 1- $p_{1}$. Matrix of transition probability of three-state Markov chain can be written as Eq.(5):

$$
P=\left[\begin{array}{lll}
P_{11} & P_{12} & P_{13} \\
P_{21} & P_{22} & P_{23} \\
P_{31} & P_{32} & P_{33}
\end{array}\right]=\left[\begin{array}{ccc}
0 & 1-P_{1} & P_{1} \\
\left(1-P_{2}\right) / 2 & P_{2} & \left(1-P_{2}\right) / 2 \\
P_{1} & 1-P_{1} & 0
\end{array}\right] .
$$

According to matrix of transition probability of three-state Markov chain,simulation model of three-state Markov chain that is derived in the MATLAB/SIMULINK software platform is shown in Fig.4. 


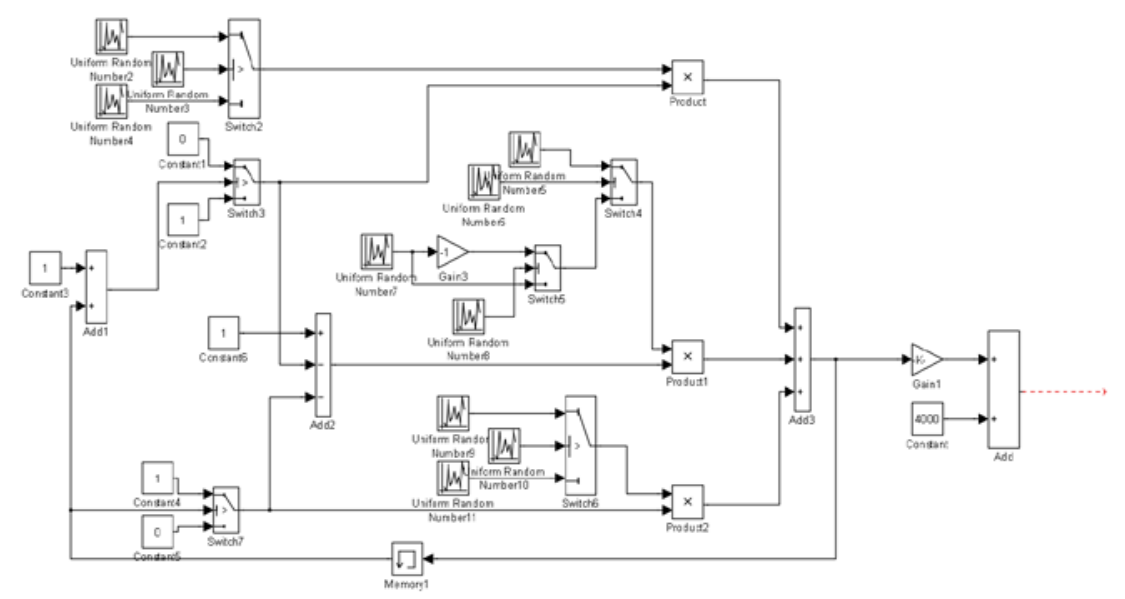

Fig. 4. Simulation model of three-state Markov chain

\section{Random SVPWM Technique base on Markov Chain Technique}

Markov chain algorithm is added to variable-frequency speed control system on basis of random switching frequency algorithm[9].Relationship between Markov chain algorithm and random switching frequency algorithm is shown in Fig.5.

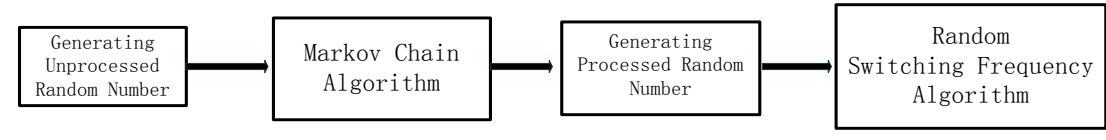

Fig. 5. Relationship between Markov chain algorithm and random switching frequency algorithm

In MATLAB/SIMULINK software platform,this paper respectively sets up simulation model of fixed switching frequency SVPWM technique,simulation model of random switching frequency SVPWM technique,simulation model of random switching frequency SVPWM technique of two-state Markov chain and simulation model of random switching frequency SVPWM technique of three-state Markov chain.Then,four kind simulation models were be respectively applied to complete simulation model of variable-frequency speed control system.

Simulation model of variable-frequency speed control system is shown in Fig.6.Model can be divided into four parts,namely part of driving signal of bridge inverter,bridge inverter,induction motor and measuring part.Above four kind simulation models were be change and implemented in the Continuous module of Fig.6,so that simulation results can be followed get.

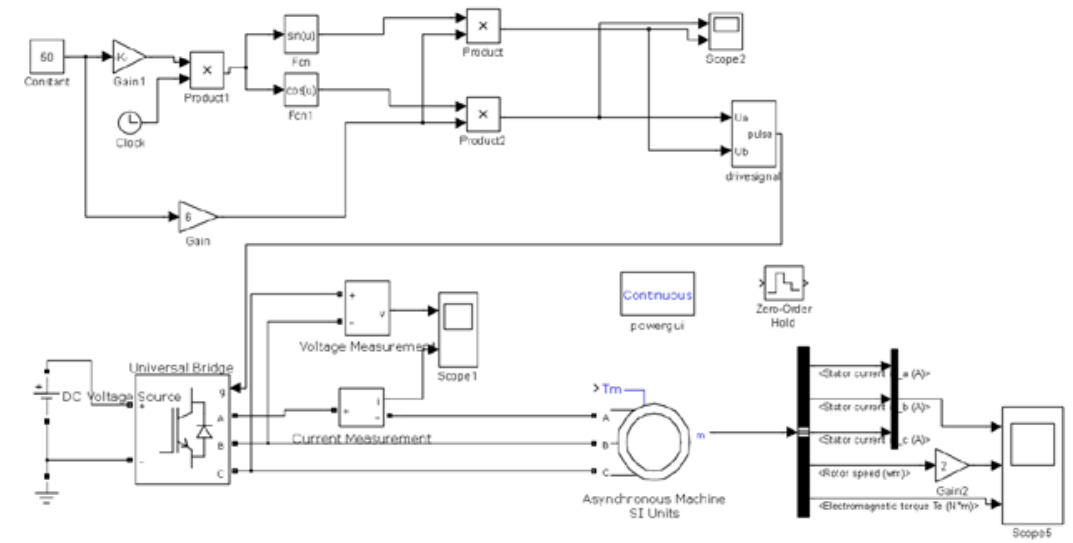

Fig. 6. Simulation model of variable-frequency speed control system

\section{Comparison and Analysis of Experimental Simulation Results}

Experimental simulation results of variable-frequency speed control system is shown in Fig.7. Fig. 7(a) and Fig.7(b) are Fast Fourier Transform Algorithm (FFT) waveform of current that are outputted 
by fixed switching frequency SVPWM technique when switching frequency are $4 \mathrm{k}$ or $8 \mathrm{k}$ respectively.Fig.7(c) and Fig.7(d) are FFT waveform of current that are outputted by random switching frequency SVPWM technique when switching frequency are 4k or 8k respectively.Fig.7(e) and Fig.7(f) are FFT waveform of current that are outputted by random switching frequency SVPWM technique of two-state Markov chain when switching frequency are $4 \mathrm{k}$ or $8 \mathrm{k}$ respectively.Fig.7(g) and Fig.7(h) are FFT waveform of current that are outputted by random switching frequency SVPWM technique of three-state Markov chain when switching frequency are $4 \mathrm{k}$ or $8 \mathrm{k}$ respectively.

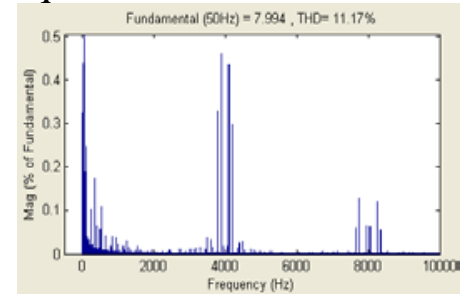

(a) FFT waveform of 4k fixed switching frequency

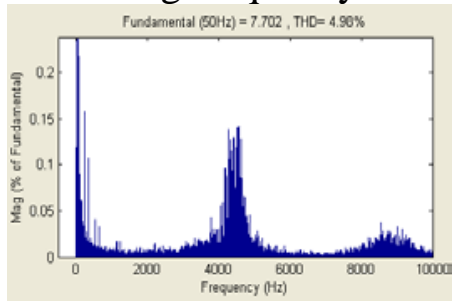

(c) FFT waveform of 4k random switching frequency

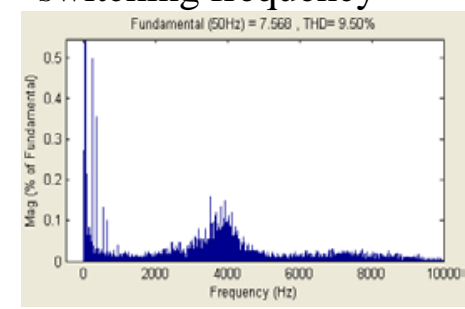

(e) FFT waveform of 4k random switching frequency of two-state Markov chain

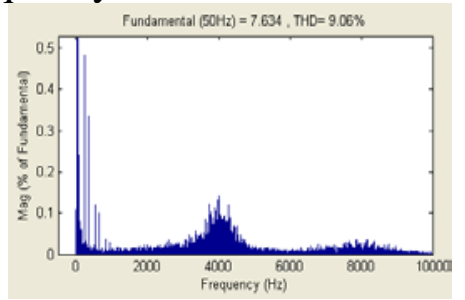

(g) FFT waveform of 4k random switching (h) frequency of three-state Markov chain

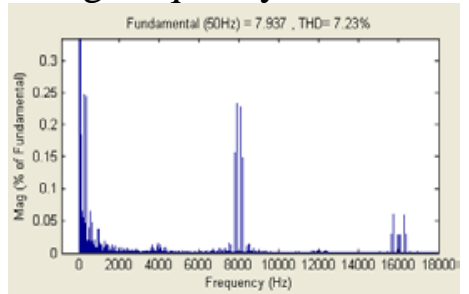

(b) FFT waveform of 8k fixed switching frequency

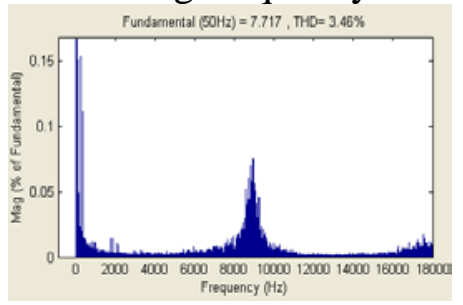

(d) FFT waveform of 8k random switching frequency

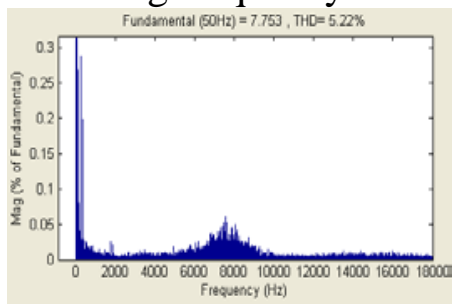

(f) FFT waveform of 8k random switching frequency of two-state Markov chain

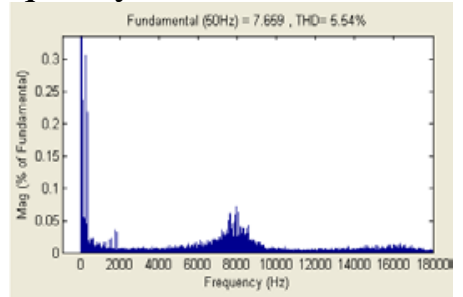

(h) FFT waveform of 8k random switching frequency of three-state Markov chain

Fig. 7. Experimental simulation results of variable-frequency speed control system

Here are some observations from Fig.7(a) and Fig.7(b).Frequency spectrum of harmonic current discretely distributes on whole frequency band,and harmonic amplitude is relatively high at integral multiple of switching frequency.This is main reason for harsh noise.In order to solve these problems, researchers put forward a method that random switching frequency SVPWM technique is applied to variable-frequency speed control system.Simulation results are illustrated in Fig.7(c) and Fig. 7(d).Simulation result can be seen that frequency spectrum of discrete harmonic current is transformed into uniform and continuous frequency spectrum,and harmonic amplitude is greatly decreased by comparing simulation result of Fig.7(a) and Fig.7(b).Although random switching frequency SVPWM technique is a feasible method,this solution also has some problems in real application.Problem that will inevitably produce multiple random numbers that are consecutive greater than mathematical expectation or consecutive less than mathematical expectation can make harmonic amplitude still maintain high.Regarding of these problems,paper proposes a more effective 
method that is random switching frequency SVPWM technique of Markov chain. Simulation results of two-state Markov chain technique are illustrated in Fig.7(e) and Fig.7(f). Simulation results can be seen that frequency spectrum of discrete harmonic current is transformed into more uniform and more continuous frequency spectrum and harmonic amplitude is greatly decreased by comparing simulation results of Fig.7(c) and Fig.7(d).Simulation results of three-state Markov chain technique are illustrated in Fig.7(g) and Fig.7(h).Simulation results can be seen that frequency spectrum of discrete harmonic current is transformed into more uniform and more continuous frequency spectrum and harmonic amplitude is greatly decreased by comparing simulation results of Fig.7(e) and Fig.7(f).Random switching frequency SVPWM technique of Markov chain decreases harmonic amplitude and solves problems of random number generation.So that Markov chain technique is correct and feasible.

\section{Conclusion}

Through experimental results, we can get these following conclusions.Markov chain technique that is added to variable-frequency speed control system reduces harmonic amplitude of output current greatly and makes local waveform of output current become more perfect.So that Markov chain technique that is added to variable-frequency speed control system technique is far superior to random variable-frequency speed control system and fixed variable-frequency speed control system.With increasing state of Markov chain,these problems of variable-frequency speed control system have been improved very much.So experimental results can prove that infinite state Markov chain technique that is applied in variable-frequency speed control system is feasible.

\section{References}

[1] A. Gupta,A. Khambadkone,"A simple space vector PWM scheme to operate a ohree level NPC inverter at high modulation index including overmodulation region,With neutral point balancing,"IEEE Transactions on Industry Applications ,vol. 43, pp.751-760, May.-Jun. 2007.

[2] A. Nabae,I.Takahashi,and H.Akagi,"A new neutral-point clamped pwm inverter," IEEE Trans. Ind. Application, vol. IA-17, pp. 518-523, Sept.-Oct. 1981.

[3] Mondai S.K.,Bose B.K.,Oleschuk V.,and Pinto J.O.P.,"Space vector pulse width modulation of three-level inverter extending operation into overmodulation region,"IEEE Transaction on Power Electronics,vol.18,no.2,pp.604-611,March 2003.

[4] M.Saeedifard,A.R.Bakhshai,G.Joos, and P.Jain,"Extending the operating range of the neurocomputing vector classification space vector modulation algorithm of three-level inverters into overmodulation region,"Industry Appl.Conf.,vol.1,pp.672-677,October 2003.

[5] K.A.Almarri and J.C.Balda,"A simple multi-level linear congruential pseudorandom number generator with a large period length,"The Fifth Joint Conference on Information Sciences (JCIS 2000),vol.1,pp.457-460,2000.

[6] Konstantin Borisov,Thomas E.Calvert,John A.Kleppe, Elaine Martin,Andrzej M.Trzynadlowski, "Experimental Investigation of a Naval Propulsion Drive Model With the PWM-Based Attenation of the Acoustic and Electromagnetic Noise,"IEEE Transactions on Industrial Electronics,vol.53,no.2, pp.450-457,APRIL 2006.

[7] P.S.Chen and Y.S.Lai,"Effective EMI filter design method for three-phase inverter based upon software noise separation",IEEE Trans. Power Electron.,vol.25,no.11,pp.2797-2806 2010.

[8] Na,S.H.;Jung,Y.G.;Lim,Y.C.;Yang,S.H.,"Reduction of audible switching noise in induction motor drive using random position space vector PWM,"Electric Power Applications,IEEE Proceedings,vol. 149,no.3,pp.195-200,May 2002.

[9] D.Jiang,R.Lai,F.Wang,F.Luo,S.Wang and D.Boroyevich,"Study of conducted EMI reduction for three-phase active front-end rectifier,"IEEE.Trans.Power Electron.,vol. 26,no.12,pp.3823-3831, 2011. 